\title{
Long-Range Spin Currents with Chiral Crystals
}

\author{
Chiral crystals can produce spin-polarized currents that propagate over \\ tens of micrometers-a promising feature for application in spintronics \\ devices.
}

By Marric Stephens

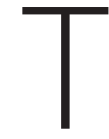

he spin-polarized currents used in spintronics are typically generated in ferromagnetic materials or materials with strong spin-orbit interaction. Spin-polarized electron currents can also be produced by certain materials whose structures have a "handedness"-an effect known as chirality-induced spin selectivity (CISS). Now, a team has shown that two chiral crystals, niobium silicide $\left(\mathrm{NbSi}_{2}\right)$ and tantalum silicide $\left(\mathrm{TaSi}_{2}\right)$, can produce and transport spin-polarized currents over distances exceeding 10 micrometers $(\mu \mathrm{m})-$ a record for this class of materials [1].

In 2020, Yoshihiko Togawa, of Osaka Prefecture University, Japan, and colleagues demonstrated CISS in a chiral crystal by showing that a charge current injected into the crystal would become spin polarized and would maintain that polarization over a distance of $1 \mu \mathrm{m}$. Such a distance was surprising, because it was greater than the length over which spins diffuse (about $100 \mathrm{~nm}$ or less), but the researchers argued that localized

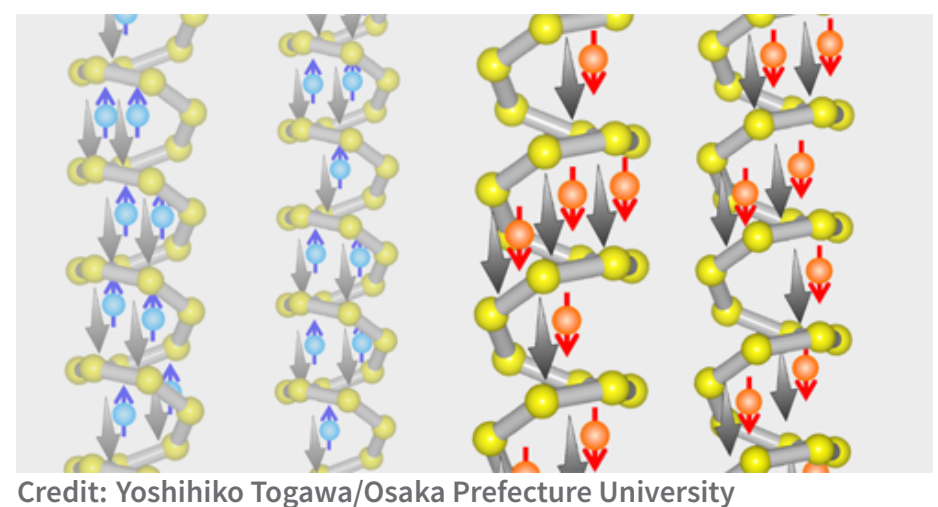

electron spins could "protect" the spins of the itinerant electrons. In the new work, Togawa's team investigated $\mathrm{NbSi}_{2}$ and $\mathrm{TaSi}_{2}$, which do not contain such localized spins. Thus, the team expected spin currents to survive over shorter distances but surprisingly found spin polarization persisting up to $10 \mu \mathrm{m}$. This distance is still shorter than that seen in graphene or silicon, but chiral crystals have the advantage of acting both as spin polarizers and spin conductors-which could allow simpler designs for spintronic devices.

The researchers say that the precise mechanism behind this long-range CISS effect is still mysterious. However, they rule out that the enhancement is due to conventional spin-orbit interaction, since $\mathrm{NbSi}_{2}$, with a weaker spin-orbit interaction than $\mathrm{TaSi}_{2}$, exhibited the strongest CISS.

Marric Stephens is a Corresponding Editor for Physics based in Bristol, UK.

\section{REFERENCES}

1. K. Shiota et al., "Chirality-induced spin polarization over macroscopic distances in chiral disilicide crystals," Phys. Rev. Lett. 127, 126602 (2021). 